05

\title{
Электромагнитно индуцированная прозрачность коротких слабых и мощных импульсов лазерного излучения
}

\author{
(C) О.М. Паршков \\ Саратовский государственный технический университет имени Гагарина Ю.А., \\ 410054 Саратов, Россия \\ e-mail: oparshkov@mail.ru
}

Поступила в редакцию 05.11.2020 г.

В окончательной редакции 09.12.2020 г.

Принята к публикации 15.12.2020 г.

\begin{abstract}
Сообщены выводы теоретического исследования зависимости характеристик явления электромагнитно индуцированной прозрачности от мощности импульса входного пробного излучения при эллиптической поляризации лазерных полей. Анализ проведен для $\Lambda$-схемы неоднородно уширенных квантовых переходов между уровнями ${ }^{3} P_{0},{ }^{3} P_{1}^{0}$ и ${ }^{3} P_{2}$ изотопа ${ }^{208} \mathrm{~Pb}$. Рассмотрены случаи резонанса, т. е. совпадения частот пробного и управляющего полей с центральными частотами соответствующих квантовых переходов, и квазирезонанса, когда такое совпадение имеет место лишь с точностью до ширины линии соответствующего квантового перехода. Показано, что в указанных случаях существует тенденция распада входного пробного излучения на импульсы с различными поляризационными характеристиками. Если входное пробное излучение достаточно слабо, то эти импульсы имеют постоянные поляризационные характеристики и являются импульсами нормальных мод. В случае большей мощности входного пробного излучения импульсы, на которые оно распадается в среде, не являются импульсами нормальных мод, но их поляризационные характеристики колеблются около значений, присущих нормальным модам. При этом фазовая модуляция пробного поля присутствует на всех стадиях его распространения в среде. Показано, что с ростом интенсивности входного пробного излучения прозрачность среды для пробного поля уменьшается. Однако она достаточно велика, если поляризационные характеристики входного пробного излучения совпадают с таковыми для нормальной моды, большая ось эллипса поляризации которой параллельна большой оси эллипса поляризации управляющего поля.
\end{abstract}

Ключевые слова: электромагнитно индуцированная прозрачность, нормальные моды.

DOI: $10.21883 /$ OS.2021.04.50783.202-20

\section{1. Введение}

Явление электромагнитно индуцированной прозрачности (ЭМИП) [1-3] привлекает внимание благодаря перспективам практического использования, а также важностью изучения его особенностей для понимания процессов взаимодействия излучения с веществом. С использованием эффекта ЭМИП созданы устройства для измерения магнитного поля [4] и точного времени [5]. Эффект имеет перспективы для применения в системах телекоммуникации и квантовой памяти $[2,6,7]$. С помощью ЭМИП создаются оптические нелинейности большой величины $[3,8]$, и может быть реализовано усиление лазерного излучения без создания инверсии населенностей уровней резонансного квантового перехода [9]. Непрерывно расширяется перечень сред, в которых возможно возникновение ЭМИП. Например, исследуется протекание этого явления в коррелированных квантовых газах [10], на примесных центрах в фотонных кристаллах [11], вблизи оптического нановолокна [12]. Расширяется и перечень способов возбуждения ЭМИП. Так, в работе [13] обсуждаются результаты исследования ЭМИП в радиочастотном диапазоне, а в [14] - при условии, что пробное излучение имеет орбитальный угловой момент.

Если уровни квантовых переходов вырождены, то в явлении ЭМИП возникают эффекты, связанные с состояниями поляризации взаимодействующих полей. Теория и эксперимент по изучению воздействия постоянного магнитного поля на круговые компоненты пробного излучения описаны в работах $[15,16]$. В работах [17-19] представлены теоретические и экспериментальные результаты по исследованию сопровождающего ЭМИП двойного лучепреломления. Теория, изложенная в работе [20], показала, что пробное поле может распространяться в среде в виде двух мод с различными поляризациями.

Подавляющее большинство теоретических исследований ЭМИП, подобных выполненным в работах [17-20], исходят из приближения заданного управляющего поля, справедливого только при малых интенсивностях пробного излучения, а также ограничиваются изучением стационарного режима этого явления, возникающего при воздействии на среду непрерывных лазерных полей. Теоретические работы, описывающие ЭМИП в полях импульсных лазеров без приближения заданного управляющего излучения, используют в основном адиабатиче- 
ское приближение [21,22], адекватное описанию достаточно длительных импульсов. В [23] было показано, что это приближение не применимо в случае ЭМИП в полях импульсов малой длительности.

В настоящем сообщении представлены результаты теоретического исследования процесса ЭМИП коротких импульсов пробного излучения (с длительностью порядка наносекунды) без использования условий адиабатического приближения и приближения заданного управляющего поля. Для определенности мы рассматриваем $\Lambda$-схему неоднородно уширенных квантовых переходов между вырожденными энергетическими уровнями ${ }^{3} P_{0}$, ${ }^{3} P_{2},{ }^{3} P_{1}^{0}$ изотопа ${ }^{208} \mathrm{~Pb}$, в парах которого экспериментально наблюдалось явление ЭМИП $[24,25]$. Предполагается, что на входе в резонансную среду пробное и управляющие поля эллиптически поляризованы и их центральные частоты могут отклоняться от центральных частот неоднородно уширенных квантовых переходов. Исследования опирались на результаты работ [26,27], в которых было показано, что в приближении заданного управляющего поля пробное излучение в среде представляет собой совокупность двух нормальных мод. Главная ось эллипса поляризации (ЭП) одной из мод параллельна, а другой перпендикулярна главной оси ЭП управляющего поля. Мода первого типа именовалась в $[26,27]$ параллельной, а мода второго типа - перпендикулярной нормальной модой. Ниже представлены результаты численного исследования трех ситуаций. В первой из них полагается, что напряженность электрического поля входного пробного импульса настолько мала, что управляющее поле в среде можно считать заданным. Далее этот случай именуется случаем слабого пробного поля. Две другие ситуации, когда максимальная напряженность электрического поля входного пробного импульса составляет примерно половину напряженности электрического поля входного управляющего излучения или равна последней, именуются случаями среднего и мощного пробного полей соответственно.

\section{2. Постановка краевой задачи}

На рис. 1, $a$ представлена $\Lambda$-схема уровней изотопа ${ }^{208} \mathrm{~Pb}$, рассматриваемая в настоящем сообщении. Нижний уровень $1\left({ }^{3} P_{0}\right)$ является простым, тогда как верхний уровень $2\left({ }^{3} P_{1}^{0}\right)$ и средний уровень $3\left({ }^{3} P_{2}\right)$ трехкратно и пятикратно вырождены. Введем ортонормированный базис $\phi_{k}(k=1,2, \ldots, 9)$ из общих собственных функций операторов энергии, квадрата и проекции момента импульса на ось $z$ для изолированного атома. Пусть функция $\phi_{1}$ относиться к уровню 1 , для которого $M=0$, функции $\phi_{k}, k=2,3,4-\kappa$ уровню 2 при $M=-1,0,1$, а функции $\phi_{k}, k=5,6, \ldots, 9-$ к уровню 3 при $M=-2,-1,0,1,2$ соответственно. Положим, что $D_{1}$ и $D_{2}-$ приведенные электродипольные моменты переходов $1 \rightarrow 2$ и $3 \rightarrow 2$ соответственно. Обозначим через $\omega_{210}$ и $\omega_{230}\left(\omega_{210}>\omega_{230}\right)$ частоты этих переходов для покоящегося атома. Для учета эффекта Допплера
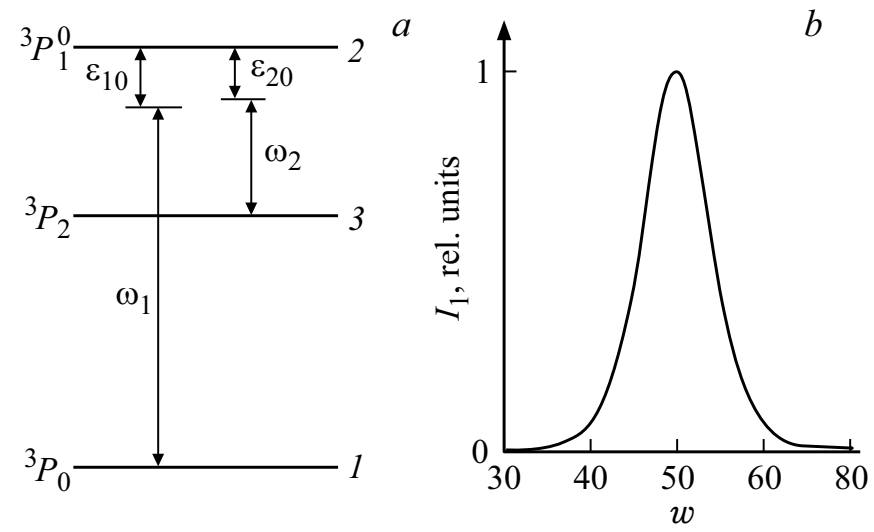

Рис. 1. $\Lambda$-схема квантовых переходов (частотные интервалы соответствуют покоящемуся атому) $(a)$; временная эволюция интенсивности $I_{1}$ входного пробного импульса $(b)$.

введем время $T_{1}=1 / \Delta_{1}$, где $\Delta_{1}-$ полуширина кривой плотности распределения частот $\omega_{21}$ квантовых переходов между уровнями 1 и 2.

Описывая лазерные поля плоскими волнами, распространяющимися вдоль оси $z$, представим полное электрическое поле в среде следующим образом:

$$
\begin{gathered}
\mathbf{E}=\mathbf{E}_{1}+\mathbf{E}_{2}, \\
\mathbf{E}_{l}=\mu_{l}\left[\mathbf{i} \tilde{E}_{x l} \cos \left(\omega_{l} t-k_{l} z+\tilde{\delta}_{x l}\right)\right. \\
\left.+\mathbf{j} \tilde{E}_{y l} \cos \left(\omega_{l} t-k_{l} z+\tilde{\delta}_{y l}\right)\right], \quad l=1,2 .
\end{gathered}
$$

Здесь $\mathbf{E}_{l}$ и $\omega_{l}-$ напряженность электрического поля и несущая частота пробного $(l=1)$ и управляющего $(l=2)$ излучений, $k_{l}=\omega_{l} / c, \mathbf{i}, \mathbf{j}-$ единичные векторы осей $x$ и $y, \tilde{E}_{x l}, \tilde{E}_{y l}$ - действительные амплитуды, а $\tilde{\delta}_{x l}$, $\tilde{\delta}_{y l}-$ функции, именуемые ниже фазовыми добавками $x$ - и $y$-компонент пробного $(l=1)$ и управляющего $(l=2)$ полей. Полагается, что функции $\tilde{E}_{x l}, \tilde{E}_{y l}, \tilde{\delta}_{x l}$, $\tilde{\delta}_{y l}$ дважды непрерывно дифференцируемы как функции двух переменных. Условие квазирезонанса означает, что $\left|\omega_{210}-\omega_{1}\right| \ll \omega_{210}$ и $\left|\omega_{230}-\omega_{2}\right| \ll \omega_{230}$.

Введем в рассмотрение амплитуды левой $f_{l}$ и правой $g_{l}([28])$ круговых компонент пробного $(l=1)$ и управляющего $(l=2)$ излучений,

$$
\begin{aligned}
& f_{l}=\left[\tilde{E}_{x l} \exp \left(i \tilde{\delta}_{x l}\right)-i \tilde{E}_{y l} \exp \left(i \tilde{\delta}_{y l}\right)\right] / \sqrt{2}, \\
& g_{l}=\left[\tilde{E}_{x l} \exp \left(i \tilde{\delta}_{x l}\right)+i \tilde{E}_{y l} \exp \left(i \tilde{\delta}_{y l}\right)\right] / \sqrt{2},
\end{aligned}
$$

и произведем разложение волновой функции $\Psi$ атома в поле (1) по функциям базиса $\phi_{k}$,

$$
\begin{aligned}
\Psi= & \bar{c}_{1} \phi_{1}+\left(\sum_{k=2}^{4} \bar{c}_{k} \phi_{k}\right) \exp \left(-i \xi_{1}\right) \\
& +\left(\sum_{k=5}^{9} \bar{c}_{k} \phi_{k}\right) \exp \left[-i\left(\xi_{1}-\xi_{2}\right)\right],
\end{aligned}
$$


где $\bar{c}_{k}-$ амплитуды вероятности заселения состояний, $\xi_{l}=\omega_{l} t-k_{l} z$. Положим далее

$$
\begin{gathered}
c_{1}=p_{1} \bar{c}_{1}, \quad c_{2}=\bar{c}_{2}, \quad c_{4}=\bar{c}_{4}, \quad c_{5}=p_{2} \bar{c}_{5}, \\
c_{7}=(1 / \sqrt{6}) p_{2} \bar{c}_{7}, \quad c_{9}=p_{2} \bar{c}_{9},
\end{gathered}
$$

где $p_{l}=2 D_{l} /\left|D_{l}\right|, l=1,2$. Введем нормированные независимые переменные $s$ и $w$ :

$$
s=z / z_{0}, \quad w=(t-z / c) / T_{1} .
$$

Исходя из уравнений Максвелла и Шредингера получим тогда в первом приближении метода вращающихся полей систему уравнений

$$
\begin{aligned}
& \frac{\partial f_{1}}{\partial s}=\frac{i}{\sqrt{\pi}} \int_{-\infty}^{+\infty} c_{1} c_{2}^{*} \exp \left[-\left(\varepsilon_{1}-\varepsilon_{10}\right)^{2}\right] d \varepsilon_{1}, \\
& \frac{\partial f_{2}}{\partial s}=-\frac{i}{\sqrt{\pi}} \xi \int_{-\infty}^{+\infty}\left(c_{4}^{*} c_{9}+c_{2}^{*} c_{7}\right) \exp \left[-\left(\varepsilon_{1}-\varepsilon_{10}\right)^{2}\right] d \varepsilon_{1}, \\
& \frac{\partial g_{1}}{\partial s}=-\frac{i}{\sqrt{\pi}} \int_{-\infty}^{+\infty} c_{1} c_{4}^{*} \exp \left[-\left(\varepsilon_{1}-\varepsilon_{10}\right)^{2}\right] d \varepsilon_{1} \\
& \frac{\partial g_{2}}{\partial s}=\frac{i}{\sqrt{\pi}} \xi \int_{-\infty}^{+\infty}\left(c_{2}^{*} c_{5}+c_{4}^{*} c_{7}\right) \exp \left[-\left(\varepsilon_{1}-\varepsilon_{10}\right)^{2}\right] d \varepsilon_{1}, \\
& \frac{\partial c_{1}}{\partial w}=-i\left(f_{1} c_{2}-g_{1} c_{4}\right), \\
& \frac{\partial c_{2}}{\partial w}+i \varepsilon_{1} c_{2}=-\frac{i}{4}\left(f_{1}^{*} c_{1}+g_{2}^{*} c_{5}-f_{2}^{*} c_{7}\right)-\gamma c_{2}, \\
& \frac{\partial c_{4}}{\partial w}+i \varepsilon_{1} c_{4}=\frac{i}{4}\left(g_{1}^{*} c_{1}-g_{2}^{*} c_{7}+f_{2}^{*} c_{9}\right)-\gamma c_{4}, \\
& \frac{\partial c_{5}}{\partial w}+i\left(\varepsilon_{1}-\varepsilon_{2}\right) c_{5}=-i g_{2} c_{2} \\
& \frac{\partial c_{7}}{\partial w}+i\left(\varepsilon_{1}-\varepsilon_{2}\right) c_{7}=\frac{i}{6}\left(f_{2} c_{2}-g_{2} c_{4}\right), \\
& \frac{\partial c_{9}}{\partial w}+i\left(\varepsilon_{1}-\varepsilon_{2}\right) c_{9}=i f_{2} c_{4} .
\end{aligned}
$$

Здесь введены следующие обозначения

$$
\begin{gathered}
\varepsilon_{1}=\left(\omega_{21}-\omega_{1}\right) / \Delta_{1}, \quad \varepsilon_{2}=\varepsilon_{20}+\beta\left(\varepsilon_{1}-\varepsilon_{10}\right), \\
\xi=0.6 \beta\left|D_{2} / D_{1}\right|^{2}, \quad \beta=\omega_{230} / \omega_{210},
\end{gathered}
$$

$\gamma=T_{1} /(2 \tau), \tau$ - время жизни уровня ${ }^{3} P_{1}^{0}$ относительно радиационного распада. Величины $\varepsilon_{10}$ и $\varepsilon_{20}$ ниже именуются отстройками резонанса, определяются формулами

$$
\varepsilon_{10}=\left(\omega_{210}-\omega_{1}\right) / \Delta_{1}, \quad \varepsilon_{20}=\left(\omega_{230}-\omega_{2}\right) / \Delta_{1}
$$

и описывают отклонения несущих частот от условий резонанса.
Ниже используются величины $a_{l}, \alpha_{l}, \gamma_{l}$ - параметры ЭП пробного $(l=1)$ и управляющего $(l=2)$ излучений: $a_{l}-$ большая полуось ЭП, измеренная в единицах $\mu_{l}, \alpha_{l}$ - угол (в радианах) между ней и осью $x, \gamma_{l}$ - параметр, именуемый ниже параметром сжатия $\left(0 \leq \alpha_{l} \leq \pi,-1 \leq \gamma_{l} \leq 1\right)$. Отметим, что $\left|\gamma_{l}\right|$ равен отношению малой оси ЭП к его большой оси и $\gamma_{l}<0 \quad\left(\gamma_{l}>0\right)$, если поляризация правая (левая) эллиптическая. Величины $\alpha_{l}$ и $\gamma_{l}$ далее называются поляризационными параметрами излучения.

Для удобства представления результатов расчета функции $\tilde{E}_{u l}, \tilde{\delta}_{u l}(u=x, y)$ в формулах (1) запишем следующим образом:

$$
\tilde{E}_{u l}=E_{u l} \exp \left(i \varphi_{u l}\right), \quad \tilde{\delta}_{u l}=\delta_{u l}+\varphi_{u l} .
$$

Здесь $E_{u l}=\left|\tilde{E}_{u l}\right|, \varphi_{u l}=0$ при $\tilde{E}_{u l}>0$ и $\varphi_{u l}=-\pi$ при $\tilde{E}_{u l}<0$. Фазовая добавка $\delta_{x l}\left(\delta_{y l}\right)$ изменяет скачком величиной $\pm \pi$ свое значение в узловых точках, т.е. в таких точках $(s, w)$, в которых величина $\tilde{E}_{x l}\left(\tilde{E}_{y l}\right)$ меняет знак. Вне этих точек величина $\delta_{x l}\left(\delta_{y l}\right)$ остается непрерывной. Отметим, что определение поля формулой (1) равносильно заданию функций $a_{l}, \alpha_{l}, \gamma_{l}$, а также одной из величин $\delta_{x l}$ и $\delta_{y l}$.

Система уравнений (2) дополнялась граничными условиями (5)

$$
\begin{gathered}
a_{l}=a_{l 0}, \quad \alpha_{l}=\alpha_{l 0}, \quad \gamma_{l}=\gamma_{l 0}, \quad \delta_{x l}=\delta_{x l 0} ; \\
s=0, \quad w \geq 0 .
\end{gathered}
$$

Здесь $a_{l 0}, \alpha_{l 0}, \gamma_{l 0}, \delta_{x l 0}$ - зависящие от $w$ функции, описывающие временное поведение величин $a_{l}, \alpha_{l}, \gamma_{l}, \delta_{x l}$ на входной поверхности $(s=0)$ среды. В качестве начальных условий полагалось, что при $w=0$ все атомы находятся на энергетическом уровне 1 рассматриваемой $\Lambda$-схемы и пробное поле внутри среды отсутствует.

При обсуждении результатов расчетов используется интенсивность $I_{l}$ пробного $(l=1)$ и управляющего $(l=2)$ излучений, измеряемых в единицах $c \mu_{1}^{2} /(8 \pi)$. Прозрачность $T r$ среды для пробного поля задается формулой $\operatorname{Tr}=W(s) / W(0)$, где $W(0)$ и $W(s)$ - количество энергии, переносимое пробным излучением через единицу площади поперечного сечения на входной поверхности $(s=0)$ и на расстоянии $s$ от нее соответственно.

\section{3. Нормальные моды}

Если пробное излучение отсутствует, то управляющее поле распространяется в среде без поглощения, поскольку уровни резонансного ему перехода не заселены. Поэтому характеристики этого поля в произвольной точке среды с точностью до временного сдвига совпадают с таковыми на входной поверхности. Если напряженность электрического поля пробного излучения значительно меньше напряженности электрического поля управляющего излучения, то допустимо приближение 
заданного управляющего поля. В этом приближении управляющее излучение распространяется в среде как в пустом пространстве. В [26,27] показано, что тогда пробное поле в среде, описываемое решением краевой задачи (2)-(5) и именуемое далее полным пробным полем, является суммой параллельной и перпендикулярной нормальных мод. Величины $\gamma_{1}$ нормальных мод постоянны во времени и пространстве, равны по модулю, но имеют разные знаки. Моды не взаимодействуют между собой, а сумма их интенсивностей совпадает с интенсивностью полного пробного поля в любой пространственно-временной точке. Способ нахождения граничных условий для каждой нормальной моды исходя из параметров полного входного пробного поля изложен в [26]. Используя эти граничные условия и систему уравнений (2), можно исследовать распространение в среде отдельной нормальной моды.

Нормальные моды имеют различные групповые скорости [27]. В случае, если полный входной пробный импульс имеет колоколообразную форму, внутри среды он разделяется на несколько импульсов, каждый из которых переносит энергию одной из нормальных мод. При этом импульсы параллельной нормальной моды распространяются с большей скоростью, чем перпендикулярной.

\section{4. Численные результаты}

При численном решении задачи (2)-(5) полагалось $\beta=0.7, \xi=2.11, \gamma=1.5 \cdot 10^{-2}$ [29]. В случае резонанса $\varepsilon_{10}=\varepsilon_{20}=0$, а в случае квазирезонанса полагалось $\varepsilon_{10}=-0.3$, а $\varepsilon_{20}=0.6$. Граничные условия (5) для импульса пробного излучения $(l=1)$ конкретизировались в виде

$$
\begin{gathered}
a_{10}=a_{m} \operatorname{sech} \frac{w-50}{5}, \quad \alpha_{10}=\frac{\pi}{6}, \\
\gamma_{10}=-0.5, \quad \delta_{x 10}=0,
\end{gathered}
$$

а для управляющего поля $(l=2)$ в виде

$$
a_{20}=6.65, \quad \alpha_{20}=0, \quad \gamma_{20}=-0.3, \quad \delta_{x 20}=0 .
$$

Равенства (6) задают входной импульс пробного излучения с колоколообразной зависимостью интенсивности $I_{1}$ от времени $w$ (рис. $\left.1, b\right)$. Равенства (7) описывают входное управляющее излучение, интенсивность которого $I_{2}$ не меняется в течение всего процесса взаимодействия волн. Такая модель соответствует схеме контринтуитивного наложения управляющего поля, обычно используемой при экспериментальном изучении явления ЭМИП [1]. Длительность входного пробного импульса по уровню полувысоты огибающей $I_{1}$ составляет 8.8 единиц времени $w$. Величина $a_{m}$ в (6) последовательно полагалась равной 0.2, 2.0 и 3.6. Реализации каждой из этих возможностей именуются ниже случаями слабого, среднего и мощного пробного полей соответственно. Отметим, что отношение максимального значения напряженности электрического поля входного пробного импульса к напряженности входного управляющего излучения составляет 0.06, 0.6 и 1.0 в случаях слабого, среднего и мощного пробного полей соответственно.

Значения параметров в граничных условиях (6), (7) выбраны с ориентацией на среду в виде насыщенных паров изотопа ${ }^{208} \mathrm{~Pb}$ при температуре около 1000 К. При такой температуре $N=1.1 \cdot 10^{14} \mathrm{~cm}^{-3}$ [30], $T_{1}=1.6 \cdot 10^{-10} \mathrm{~s}, z_{0}=0.01 \mathrm{~cm}$. Использованный в $(6)$ вид функции $a_{10}$ соответствует входному пробному импульсу длительностью $1.4 \mathrm{~ns}$ по уровню полувысоты огибающей его интенсивности. Заданное в (7) значение $a_{20}$ соответствует интенсивности $20 \mathrm{~kW} / \mathrm{cm}^{2}$ входного управляющего излучения. Примерно такой она была в экспериментальных работах [24,25]. Отметим, что величину интенсивности $\hat{I}_{1}$ пробного поля в $\mathrm{kW} / \mathrm{cm}^{2}$ можно оценить по формуле $\hat{I}_{1}=1.3 I_{1}$.

\section{1. Слабое пробное поле}

На рис. 2 представлены графики зависимости от $w$ величин $I_{1}, \alpha_{1}, \gamma_{1}$ для двух значений $s$. Рисунки $2, a, b$ относятся к случаю резонанса, тогда как рис. $2, c, d-$ к случаю квазирезонанса. В обоих случаях в среде происходит распад пробного импульса на несколько импульсов, каждый из которых обладает постоянными поляризационными характеристиками $\alpha_{1}, \gamma_{1}$. При $s=400$ $($ рис. $2, b, d)$ в области нахождения первого импульса $\alpha_{1}=0, \quad \gamma_{1}=0.742$, а в области нахождения других импульсов $\alpha_{1}=\pi / 2, \gamma_{1}=-0.742$. Оценки, проведенные по методике работы [26], показывают, что именно такие значения поляризационных параметров должны иметь излучения параллельной и перпендикулярной нормальных мод соответственно.

Расчеты, описывающие эволюцию каждой нормальной моды в отдельности, показали, что на всех расстояниях $s$ внутри среды выполняется условие $I_{1}=I_{1}^{(1)}+I_{1}^{(2)}$, где $I_{1}^{(1)}$ и $I_{1}^{(2)}-$ интенсивности параллельной и перпендикулярной нормальных мод соответственно. Следовательно, в первом импульсе на рис. $2, b, d$ сосредоточена энергия параллельной нормальной моды, а в остальных - перпендикулярной.

В [26] показано, что импульсы нормальных мод в условиях резонанса не обладают фазовой модуляцией. Данное обстоятельство имеет место и в рассматриваемом случае. При квазирезонансе, как показывают расчеты, ситуация меняется. На рис. 3 представлены фрагменты зависимости фазовых добавок $\delta_{x 1}$ и $\delta_{y 1}$ от $w$ для двух значений расстояния $s$ в случае квазирезонанса совместно с кривыми, описывающими эволюцию величин $a_{1}$. Импульс 1 на обоих рисунках относится к параллельной нормальной моде, а импульс $2-$ к перпендикулярной. Непостоянство величин $\delta_{x 1}$ и $\delta_{y 1}$ означает наличие фазовой модуляции. В районе импульсов 1 фазовая модуляция незначительна, тогда как в районе импульса 2 она достаточно велика. 

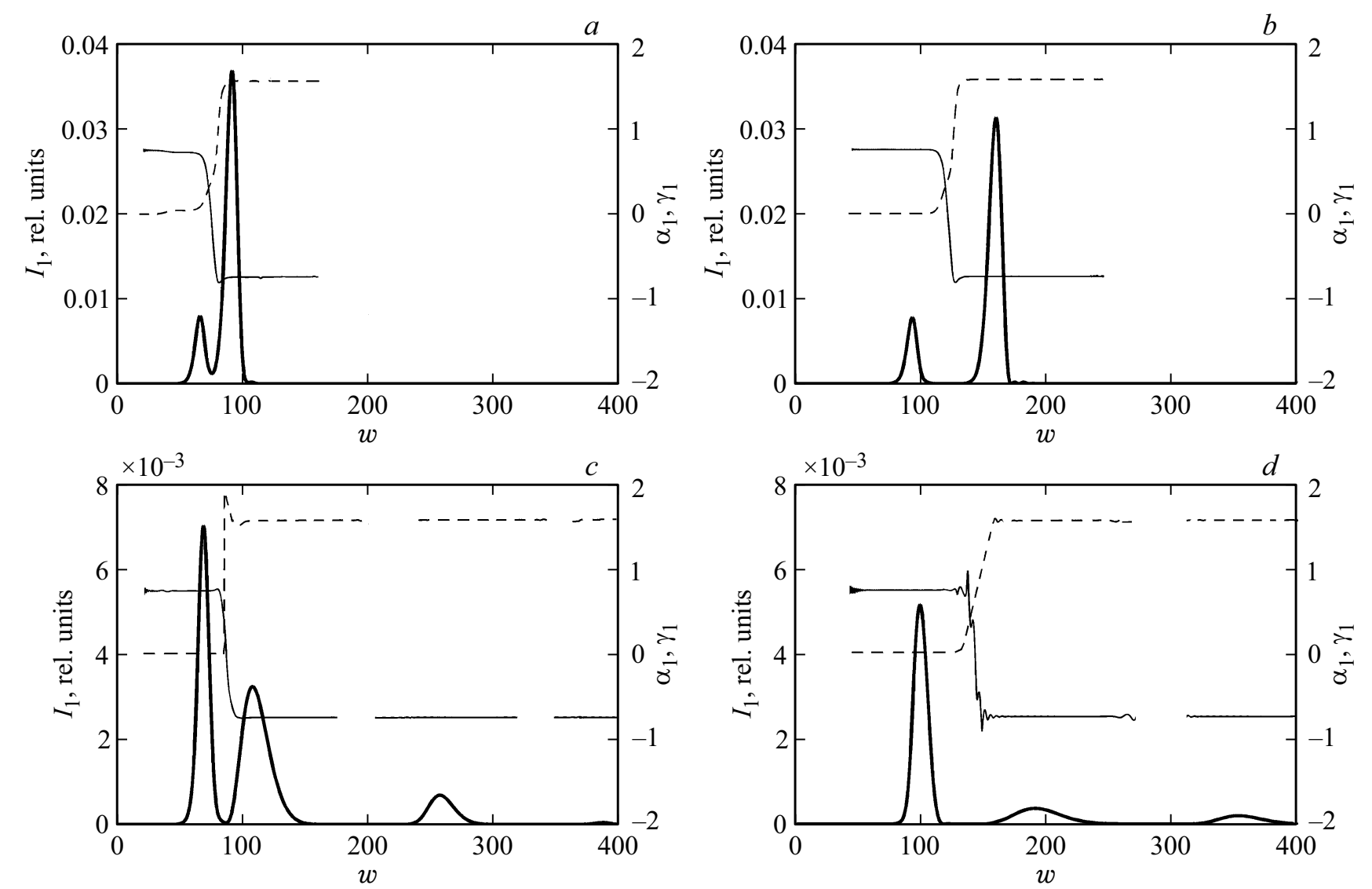

Рис. 2. Эволюция величин $I_{1}$ (толстая линия), $\alpha_{1}$ (штриховая линия), $\gamma_{1}$ (тонкая линия) в случае резонанса при $s=150$ (a) и $s=400(b)$ и квазирезонанса при $s=150(c)$ и $s=400(d)$.
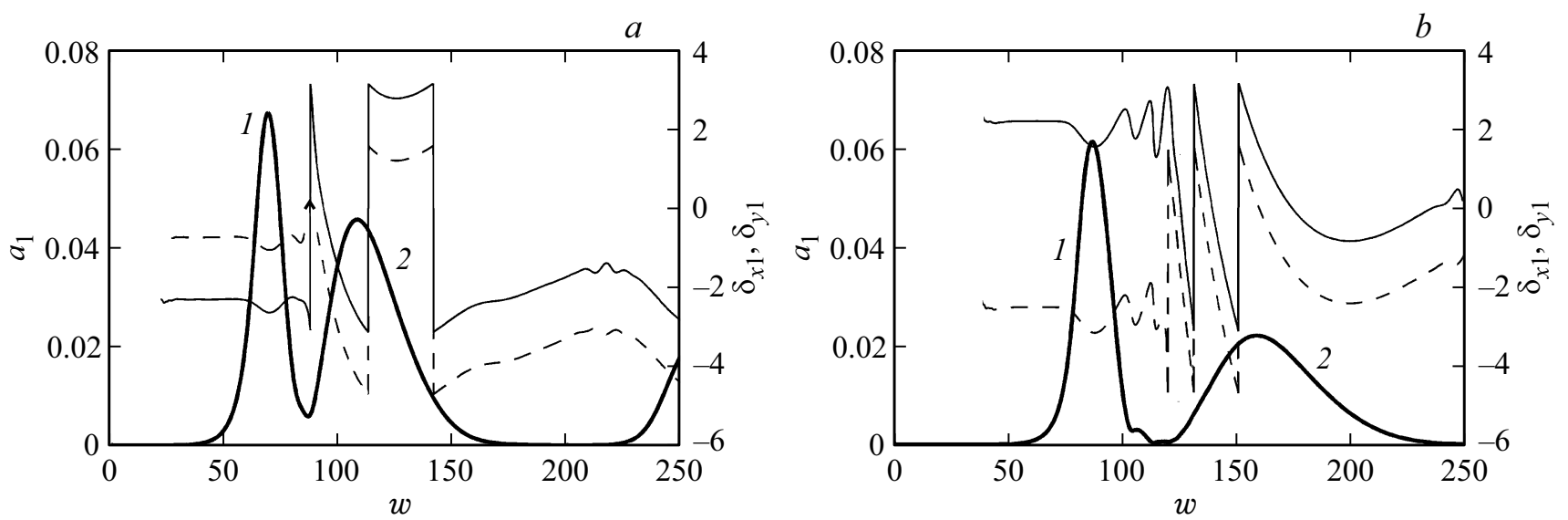

Рис. 3. Эволюция величин $a_{1}$ (толстая линия), $\delta_{x 1}$ и $\delta_{y 1}$ (тонкая и штриховая линии соответственно) при $s=150(a)$ и $s=300(b)$; 1 - параллельная, 2 - перпендикулярная нормальная моды.

Мгновенная частота пробного излучения определяется формулой

$$
\tilde{\omega}_{1}=\omega_{1}+\left(1 / T_{1}\right)\left(\partial \tilde{\delta}_{x 1} / \partial w\right) .
$$

Оценки показывают, что при $s=300$ сдвиг мгновенной частоты в районе импульса перпендикулярной нормальной моды (второй импульс на рис. 2,b) почти вдвое больше, чем ширина спектра, обусловленная длительно- стью этого импульса, т.е. является большой величиной. При этом сдвиг мгновенной частоты отрицателен, и, следовательно, мгновенная частота затягивается к резонансу с квантовым переходом $1 \rightarrow 2$.

Отметим также, что в районе импульса 1 выполняется условие $\delta_{y 1}=\delta_{x 1}+\pi / 2$, а в районе импульса $2-$ условие $\delta_{y 1}=\delta_{x 1}-\pi / 2$. Эти условия характерны для эллиптически поляризованных излучений. Они существенно 
нарушаются на малых расстояниях от входной поверхности, когда импульсы нормальных мод налагаются друг на друга, и пробное поле не является поляризованным эллиптически.

При резонансном слабом входном пробном импульсе на дистанции $s=400$ прозрачность $T r=0.95$ для полного поля, а для параллельной и перпендикулярной нормальных мод она равна 0.99 и 0.94. При отсутствии управляющего поля, как показывают расчеты, пробное излучение практически полностью поглощается средой уже на расстоянии $s=5$. Таким образом, эффективность явления ЭМИП достаточно велика.

При квазирезонансном слабом входном пробном импульсе прозрачность среды для полного поля значительно меньше: для дистанции $s=400$ она равна 0.19 , тогда как для параллельной и перпендикулярной нормальных мод величина прозрачности составляет 0.88 и 0.06 соответственно. Импульсы полного пробного поля и перпендикулярной нормальной моды теряют примерно половину своей энергии уже на расстоянии $s=60$. Отметим, что во всех рассматриваемых ситуациях величина $W(0)$ для перпендикулярной нормальной моды более чем в 5 раз больше, чем для параллельной нормальной моды. Низкая прозрачность среды для полного поля объясняется большими потерями энергии перпендикулярной нормальной моды, переносящей основную часть энергии пробного излучения. Для поля параллельной нормальной моды прозрачность среды достаточно высока.

\section{2. Среднее пробное поле}

На рис. 4 представлены графики зависимости от $w$ величин $I_{1}, \alpha_{1}, \gamma_{1}$ для двух значений $s$. Рисунки $4, a, b$ описывают процесс ЭМИП в случае резонанса. Эти рисунки свидетельствуют о том, что пробный импульс в среде распадается на два отдельных импульса. На большом расстоянии (рис. 4, $b$ ) с погрешностью менее $2 \%$ поляризационные характеристики $\alpha_{1}, \gamma_{1}$ одного из них (импульс 1) такие же, какие должны быть у параллельной нормальной моды, а в области другого (импульс 2) эти характеристики близки к соответствующим характеристикам перпендикулярной нормальной моды.

Рисунки 4, $c, d$ описывают процесс эволюции величин $I_{1}, \gamma_{1}$ при квазирезонансе. (Графики величины $\alpha_{1}$ не представлены, поскольку соответствующие кривые чрезмерно усложняют вид рисунков.) Рисунок $4, d$ показывает, что даже на расстоянии $s=600$ четкого разделения входного пробного поля на ряд изолированных импульсов не происходит. При этом в области пика 1 величина $\gamma_{1}$ осциллирует около значения, характерного для параллельной нормальной моды, а в области пика 2 - вблизи значения, характерного для перпендикулярной нормальной моды. Величина $\alpha_{1}$ колеблется, как показывает расчет, около значения 0 в области пика 1 и $\pi / 2$ в области пика 2.

Исходя из (6), (7) и используя методику работы [26], входной пробный импульс можно представить в ви- де суммы двух импульсов с поляризационными характеристиками, присущими нормальным модам. При распространении в среде по отдельности каждый из них испытывает определенную деформацию. Излучения, порожденные в среде входными полями с характеристиками параллельной и перпендикулярной нормальных мод, будем называть параллельным и перпендикулярным излучениями соответственно. Пусть теперь символ $I_{1}^{(1)}$ означает интенсивность параллельного излучения, а символ $I_{1}^{(2)}$ - интенсивность перпендикулярного излучения. На рис. 5 для расстояния $s=600$ представлены кривые интенсивностей $I_{1}^{(1)}$ и $I_{1}^{(2)}$ в случаях резонанса (рис. 5,a) и квазирезонанса (рис. 5,b) совместно с кривой $I_{1}$, описывающей эволюцию полного пробного поля.

В случае резонанса (рис. 5, $a$ ) кривая $I_{1}^{(1)}$ в районе импульса 1 незначительно отличается от кривой $I_{1}$. При этом в области нахождения импульса 2 тонкая кривая, описывающая величину $I_{1}^{(2)}$, совпадает с кривой $I_{1}$. Это в свою очередь означает приближенное выполнение условия $I_{1}=I_{1}^{(1)}+I_{1}^{(2)}$. Выше отмечалось хорошее совпадение поляризационных характеристик $\alpha_{1}, \gamma_{1}$ полного пробного поля в районе импульсов 1 и 2 с соответствующими характеристиками нормальных мод в случае резонанса. Поэтому представление о распространении пробного поля в виде суммы двух невзаимодействующих нормальных мод приближенно справедливо и в случае резонансного ЭМИП пробного излучения средней мощности.

При квазирезонансе ситуация иная. Согласно рис. $5, b$, сумма ординат пунктирной и тонкой линий при каждом фиксированном значении переменной $w$ почти всюду существенно отличается от значения ординаты толстой линии при том же значении $w$. А это означает, что условие $I_{1}=I_{1}^{(1)}+I_{1}^{(2)}$ не выполняется даже приближенно. Таким образом, в условиях квазирезонанса и пробного излучения средней мощности представление о нормальных модах теряет смысл.

Графики величин $a_{1}$ и $\delta_{x 1}$ для полного пробного поля на расстоянии $s=600$ представлены для случая резонанса на рис. $6, a$. Изменения величины $\delta_{x 1}$ в области нахождения импульсов 1 и 2 достаточно малы. Поэтому можно считать, что эти импульсы практически лишены фазовой модуляции. (Скачки величины $\delta_{x 1}$ справа от основной части импульса 2 имеют величину $\pi$ и означают смену знака величины $\tilde{E}_{x 1}$.) Следует отметить, что значения величины $\delta_{x 1}$ в областях импульса 1 и импульса 2 менее чем на $10 \%$ отличаются от тех, которые предписываются теорией [26] параллельной и перпендикулярной нормальным модам соответственно.

На рис. $6, b$ представлены графики величин $a_{1}$ и $\delta_{x 1}$ для полного пробного поля на расстоянии $s=600$ в случае квазирезонанса. Вид кривой $a_{1}$ на этом рисунке свидетельствует о том, что энергия пробного поля сосредоточена в одном импульсе довольно нерегулярной формы. Характер кривой $\delta_{x 1}$ указывает на наличие 

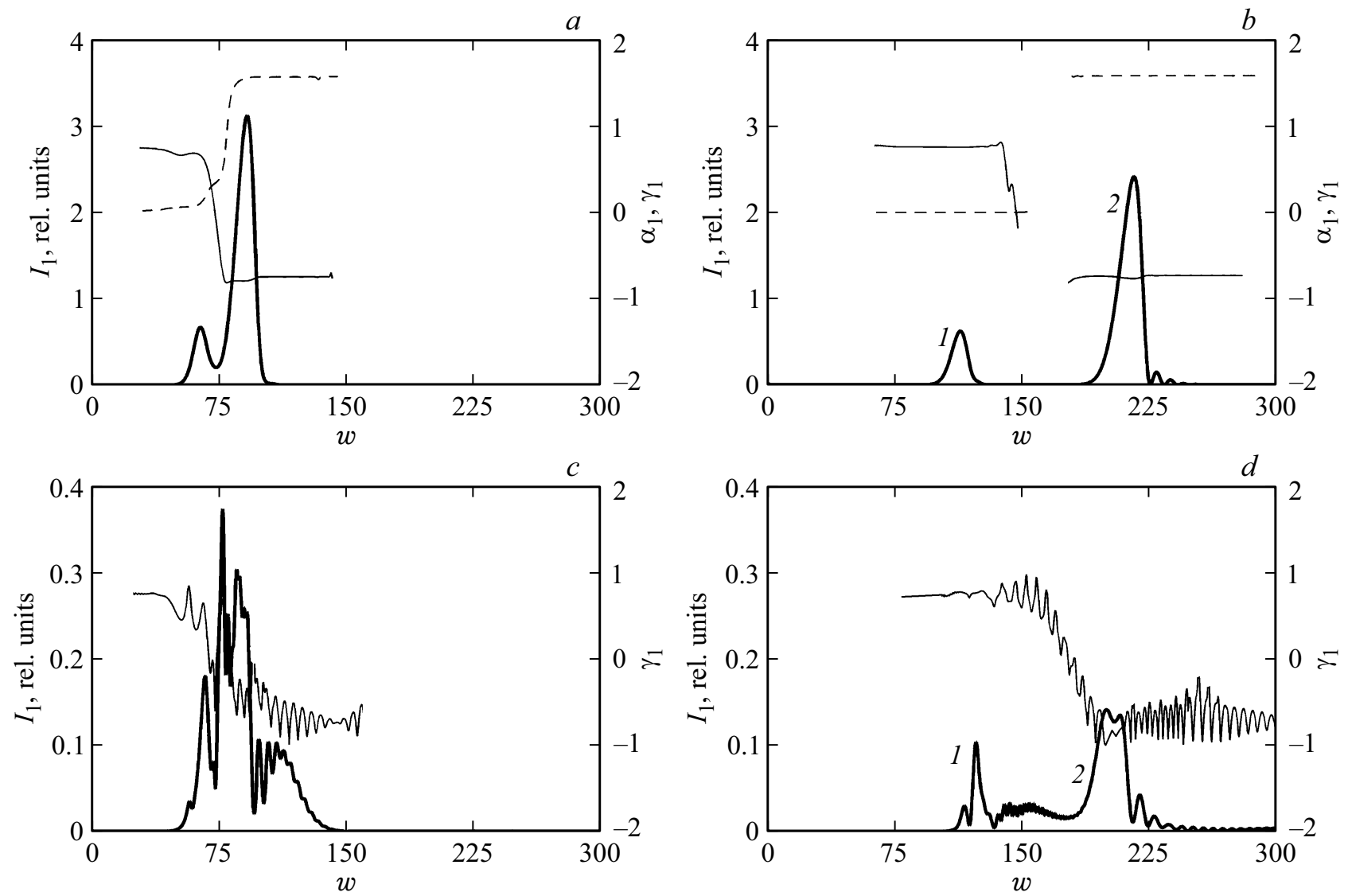

Рис. 4. Эволюция величин $I_{1}$ (толстая линия), $\alpha_{1}$ (штриховая линия), $\gamma_{1}$ (тонкая линия) в случае резонанса при $s=150(a)$ и $s=600(b)$ и величин $I_{1}$ (толстая линия), $\gamma_{1}$ (тонкая линия) при квазирезонансе для $s=150(c)$ и $s=600(d)$.
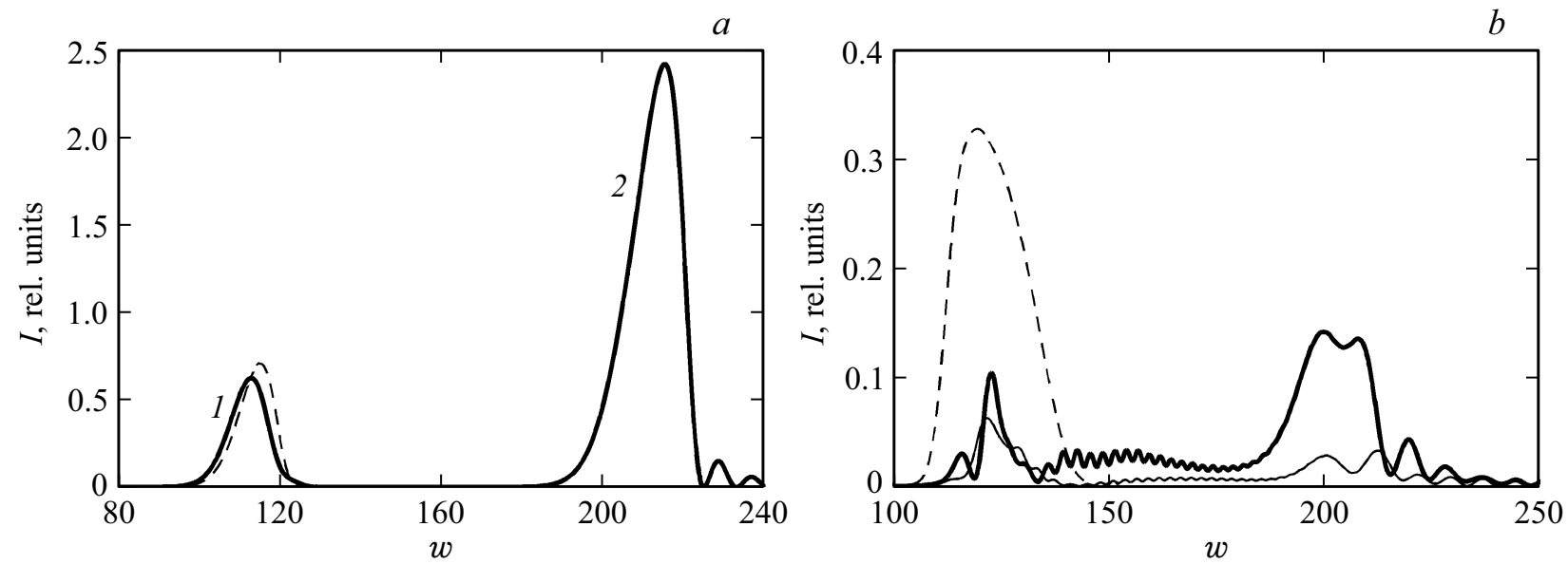

Рис. 5. Эволюция величины $I_{1}$ (толстая линия), $I_{1}^{(1)}$ (штриховая линия) и $I_{1}^{(2)}$ (тонкая линия) на расстоянии $s=600$ в случае резонанса и $(a)$ и квазирезонанса $(b)$.

фазовой модуляции этого импульса. При этом на некоторых участках импульса мгновенная частота $\tilde{\omega}_{1}$ больше, чем $\omega_{1}$, а на других - меньше. Фазовая модуляция достаточно велика. Так, например, вблизи точки $w=200$ величина $\left|\tilde{\omega}_{1}-\omega_{1}\right|$ составляет примерно 0.7 ширины линии контура неоднородного уширения квантового перехода $1 \rightarrow 2$. Это примерно в три раза больше сдвига мгновенной частоты в случае перпендикулярной моды при слабом входном пробном излучении.

В случае резонанса на расстоянии $s=400$ величина $\operatorname{Tr}$ равна 0.93, 0.99 и 0.92 для полного, параллельного и перпендикулярного излучений соответственно. При квазирезонансе для полного пробного поля значение $\mathrm{Tr}$ составляет 0.13 , а для полей параллельного и перпенди- 

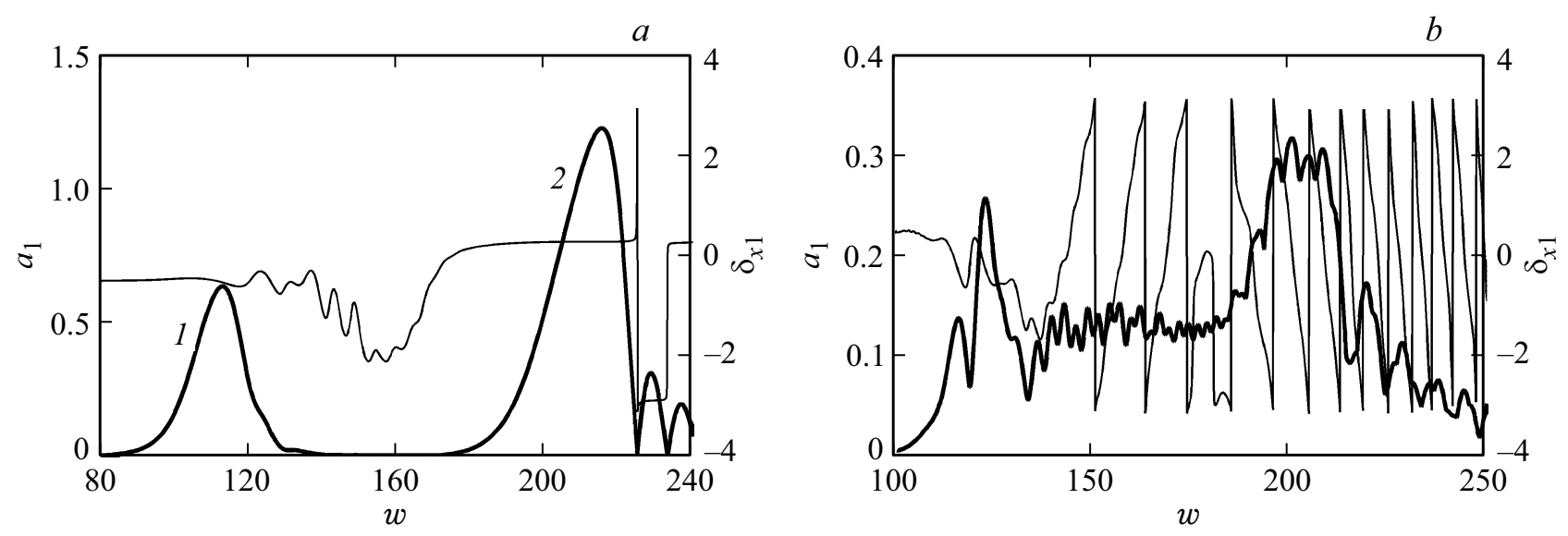

Рис. 6. Эволюция величин $a_{1}$ (толстая линия), $\delta_{x 1}$ (тонкая линия) при $s=600$ в случае резонанса $(a)$ и квазирезонанса $(b)$.

кулярного излучений 0.88 и 0.06 соответственно на том же расстоянии. При этом полное и перпендикулярные излучения теряют более половины своей энергии уже на расстоянии $s=40$, тогда как и в случае резонанса, и в случае квазирезонанса прозрачность среды для параллельного излучения достаточно высока. Выбранные для расчета параметры входного пробного излучения таковы, что при отсутствии управляющего поля на частоте пробного излучения в среде должны возникнуть пять $2 \pi$-импульсов [31], которые ввиду наличия релаксации полностью затухают на расстоянии $s=100$.

\section{3. Мощное входное пробное поле}

На рис. 7 представлены графики зависимости от $w$ величин $I_{1}, \gamma_{1}$ для двух значений величины $s$. Рисунки 7, $a, b$ относятся к процессу ЭМИП в случае резонанса, а рис. 7, $c, d-$ к процессу ЭМИП при квазирезонансе. Рисунки $7, b, d$ показывают, что на расстоянии $s=600$ пробный импульс в среде распадается на два фрагмента, отмеченные цифрами 1 и 2. В областях расположения фрагментов 1 и 2 величина $\gamma_{1}$ испытывает осцилляции около значений, близких к предписываемой теорией для параллельной и перпендикулярной нормальных мод соответственно. Аналогичное заключение относится и к эволюции величины $\alpha_{1}$, графики которой на данных рисунках не представлены.

Так же как и в случае входного пробного поля средней мощности, входной пробный импульс можно представить в виде суммы двух импульсов с характеристиками нормальных мод и изучить дальнейшую эволюцию этих импульсов. Расчеты показали, что условие $I_{1}=I_{1}^{(1)}+I_{1}^{(2)}$ существенно нарушается при распространении указанных импульсов в среде как в условиях резонанса, так и в условиях квазирезонанса.

Расчеты показывают также, что на всех расстояниях $s$ пробное излучение обладает заметной фазовой модуляцией. В частности, при больших значениях $s$ фазовая модуляция присутствует у обоих фрагментов, представленных на рис. $7, b, d$, хотя у фрагмента 2 она значительно больше, чем у фрагмента 1 .

В случае мощного резонансного входного излучения на расстоянии $s=400$ прозрачность $\operatorname{Tr}$ составляет 0.41, 0.99 и 0.42 для полного, параллельного и перпендикулярного излучений соответственно. При этом полное поле теряет половину своей энергии на расстоянии $s=130$, а перпендикулярное - на расстоянии $s=160$.

При квазирезонансном мощном пробном импульсе на расстоянии $s=400$ прозрачность $T r$ составляет 0.03, 0.88 и 0.055 для полного, параллельного и перпендикулярного излучений соответственно. При этом полное поле теряет половину своей энергии на расстоянии $s=60$, а перпендикулярное - на расстоянии $s=50$. Как в случае резонанса, так и при квазирезонансе прозрачность среды для параллельного излучения достаточно велика. В дополнение к сказанному отметим, что при отсутствии управляющего поля входной импульс полного пробного излучения в среде должен был бы породить цуг из девяти затухающих $2 \pi$-импульсов

\section{4. Прозрачность среды}

Во всех рассмотренных выше случаях отмечалось, что прозрачность среды наиболее велика для входного пробного излучения, поляризационные характеристики которого совпадают с таковыми для параллельной нормальной моды. Однако расчеты, на которых основывалось это утверждение, выполнялись в предположении, что энергия $W(0)$ параллельного излучения в пять раз меньше, чем перпендикулярного. В связи с этим для всех рассмотренных выше случаев были проделаны расчеты, описывающие эволюцию параллельного и перпендикулярного излучений при условии, что формы огибающих интенсивностей и энергии на входной поверхности одинаковы и совпадают с таковыми для полного пробного поля. Эти расчеты подтвердили вывод о том, что при одинаковых начальных условиях прозрачность для параллельного излучения больше, чем для полного и перпендикулярного. Ниже представлены 

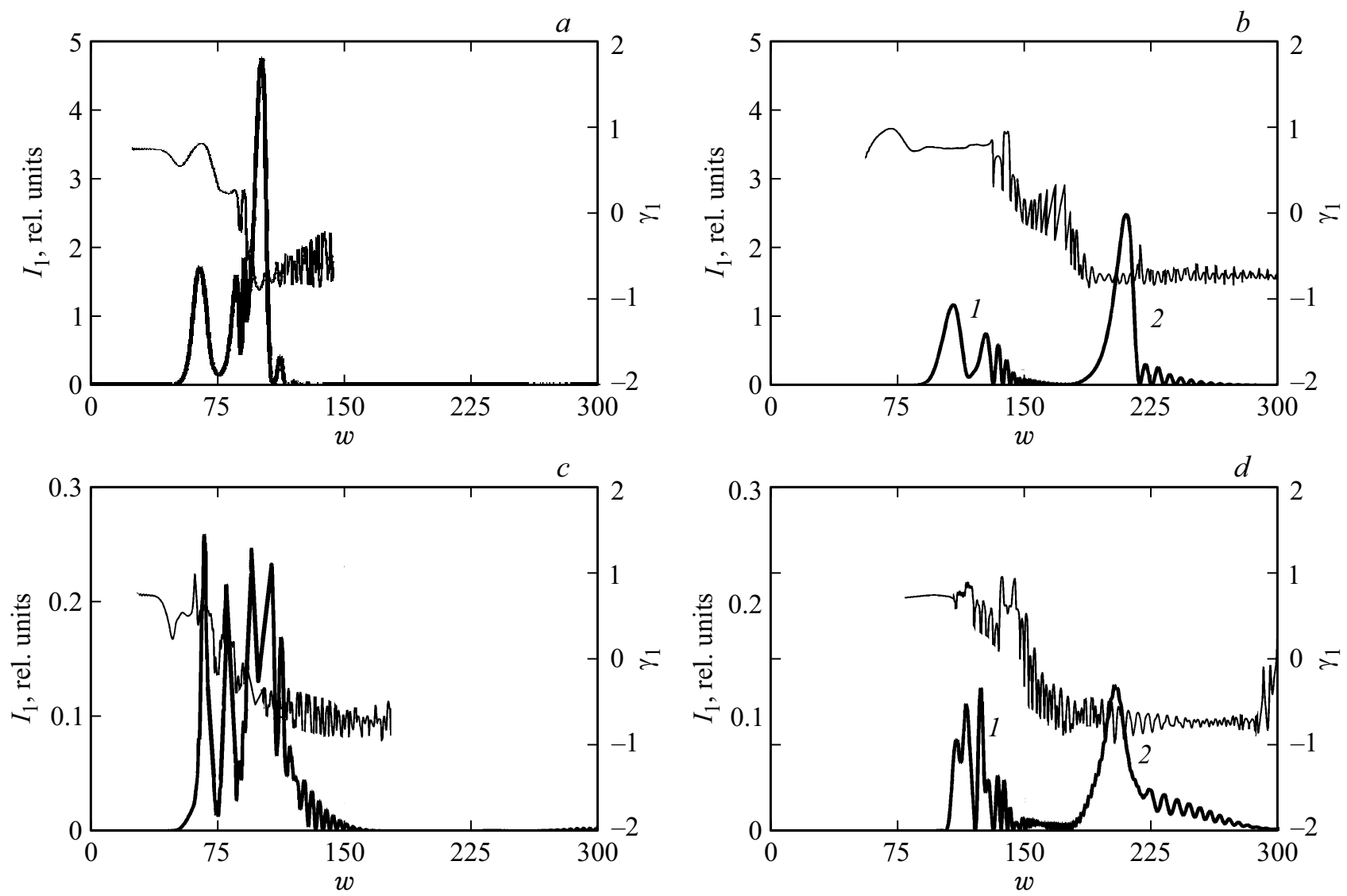

Рис. 7. Эволюция величин $I_{1}$ (толстая линия) и $\gamma_{1}$ (тонкая линия) в случае резонанса при $s=200(a)$ и $s=600$ (b) и квазирезонанса при $s=200(c)$ и $s=600(d)$.
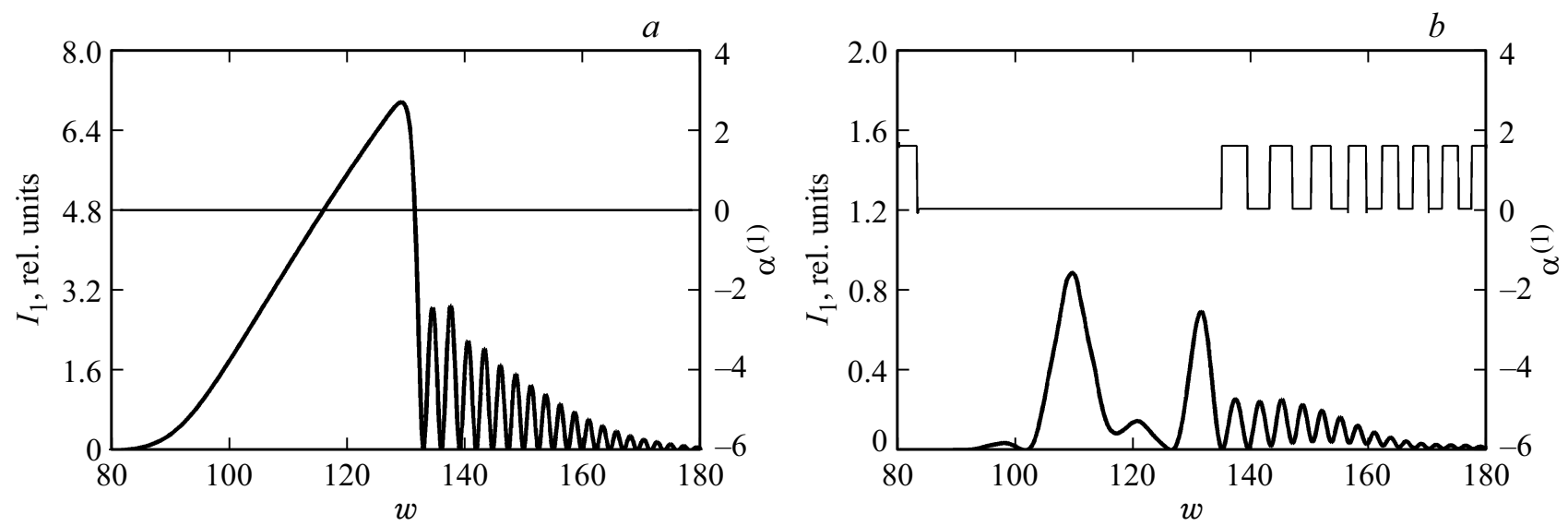

Рис. 8. Эволюция величин $I_{1}$ (толстая линия) и $\alpha^{(1)}$ (тонкая линия) при $s=600$ в случае резонанса $(a)$ и квазирезонанса $(b)$.

результаты расчета для параллельного (рис. 8, $a$ ) и перпендикулярного (рис. $8, b)$ излучений в случае мощного строго резонансного излучения при $s=600$. Символ $\alpha^{(1)}$ $\left(0 \leq \alpha^{(1)} \leq \pi / 2\right)$ использован для обозначения острого или прямого угла между линией оси $x$ и большой осью ЭП входного пробного излучения.

Сравнение кривых интенсивности $I_{1}$ на рис. 8, $a$ и рис. $8, b$, а также на рис. $7, b$ показывает, что максимальная интенсивность параллельного излучения примерно на порядок превосходит таковую для перпендикулярного излучения и более чем в три раза больше, чем для полного поля. Значение прозрачности $\operatorname{Tr}$ для параллельного излучения при $s=600$ составляет 0.94, тогда как для полного и перпендикулярного излучений на том же расстоянии она равна 0.39 и 0.07 соответственно.

Кривые $\alpha^{(1)}$ на рис. 8, $a$ показывают, что главная ось ЭП параллельного излучения в любой момент времени остается параллельной оси $x$. Рисунки $8, b$ свидетель- 
ствует о том, что главная ось ЭП перпендикулярного излучения параллельна оси $x$ на переднем фронте импульса, а на заднем фронте попеременно параллельна и перпендикулярна этой оси. Отметим, что как показывают расчёты, в случае полного квазирезонансного мощного пробного излучения угол $\alpha^{(1)}$ принимает различные значения внутри отрезка своего определения при прохождении импульсом заданной точки пространства

\section{5. Заключение}

Показано, что с увеличением мощности входного пробного импульса модовый режим распространения пробного излучения в среде не возникает. Однако при резонансе отклонение от него сравнительно невелико даже в случае, когда абсолютная величина напряженности поля входного пробного импульса достигает половины абсолютной величины напряженности поля управляющего излучения. При этом фазовая модуляция импульсов пробного поля в среде практически отсутствует. В случае квазирезонанса при таких же условиях модовый режим не имеет места, и пробное излучение обладает значительной фазовой модуляцией. Входной пробный импульс распадается в среде на фрагменты, поляризационные параметры которых колеблются около значений, свойственных нормальным модам. При мощном входном пробном поле модовый режим распространения пробного излучения отсутствует как при резонансе, так и при квазирезонансе, и в обоих случаях имеет место значительная фазовая модуляция пробного излучения.

Прозрачность среды для пробного излучения зависит от поляризационных характеристик входного пробного импульса и в общем случае существенно уменьшается с ростом интенсивности последнего. Кроме того, она уменьшается при переходе от резонансного режима ЭМИП к квазирезонансному. Однако прозрачность сохраняет достаточно высокое значение в случае, если поляризационные характеристики входного пробного импульса совпадают с теми, которые теория нормальных мод приписывает параллельной нормальной моде.

\section{Конфликт интересов}

Авторы заявляют, что у них нет конфликта интересов.

\section{Список литературы}

[1] Harris S.E. // Phys. Today. 1997. V. 50. N 7. P. 36.

[2] Lukin M.D. // Rev. Mod. Phys. 2003. V. 75. N 2. P. 457.

[3] Fleischhauer M., Imamoğlu A., Marangos J.P. // Rev. Mod. Phys. 2005. V. 77. N 2. P. 633.

[4] Martinalli M., Valente P., Failache H., Felinto D., Cruz L.S., Nussenzveig P., Lezama A. // Phys. Rev. A. 2004. V. 69. N 4. P. 043809.

[5] Godone A., Micallilizio S., Levi F. // Phys. Rev. A. 2002. V. 66. N 6. P. 063807.
[6] Duan L.-M., Lukin M.D., Cirac J.I., Zoller P. // Nature (London). 2001. V. 414. P. 413.

[7] Sinatra A. // Phys. Rev. Lett. 2006. V. 97. N 25. P. 253601.

[8] Lukin M.D., Imamoğlu A. // Nature (London). 2001. V. 413. P. 273.

[9] Kocharovskaya O., Mandel P. // Phys. Rev. A. 1990. V. 42. N 1. P. 523.

[10] Jen H.H., Daw-Wei Wang. // Phys. Rev. A. 2013. V. 87. N 6. P. $061802(\mathrm{R})$.

[11] Ronggang Liu, Tong Liu, Yingying Wang, Yujie Li, Bingzheng Gai. // Phys. Rev. A. 2017. V. 96. N 5. P. 053823.

[12] Fam Le Kien, Rauschenbeutel A. // Phys. Rev. A. 2015. V. 91. N 5. P. 053847.

[13] Basler C., Grzesiak J., Helm H. // Phys. Rev. A. 2015. V. 92. N 1. P. 013809.

[14] Hai-Hua Wang, Jing Wang, Zhi-Hui Kang, Lei Wang, Jin-Yue Gao, Yi Chen, Xiao-Jun Zhang. // Phys. Rev. A. 2019. V. 100. N 3. P. 013822.

[15] Agarwal G.S., Shubhrangshu Dosgupta.// Phys. Rev. A. 2003. V. 67. N 2. P. 023814.

[16] Sautenkov V.A., Rostovtsev Y.V., Chen H., Hsu P., Agarwal G.S., Scully M.O. // Phys. Rev. Lett. 2005. V. 94. N 23. P. 233601.

[17] Wielandy S., Gaeta A.L. // Phys. Rev. Lett. 1998. V. 81. N 16. P. 3359.

[18] Bo Wang, Shujing Li, Jie Ma, Hai Wang, Peng K.C., Min Xiao. // Phys. Rev. A. 2006. V. 73. N 5. P. 051801(R).

[19] Tai Hyun Yoon, Chang Yong Park, Sung Jong Park. // Phys. Rev. A. 2004. V. 70. N 6. P. 061803(R).

[20] Kis Z., Demeter G., Janszky J.J. // Opt. Soc. Am. B. 2013. V. 30. N 4. P. 829.

[21] Grobe R., Hioe F.T., Eberly J.H. // Phys. Rev. Lett. 1994. V. 73. N 24. P. 3183.

[22] Shakhmuratov R.N., Odeurs J. // Phys. Rev. A. 2006. V. 74. P. 043807.

[23] Kozlov V.V., Kozlova E.B. // Opt. Commun. 2009. V. 282. N 5. P. 892.

[24] Kasapi A., Maneesh Jain, Yin G.Y., Harris S.E. // Phys. Rev. Lett. 1995. V. 74. N 13. P. 2447.

[25] Maneesh Jain, Kasapi A., Yin G.Y. // Phys. Rev. Lett. 1995. V. 75. N 23. P. 4385.

[26] Париков О.М. // Квант. электрон. 2018. Т. 48. № 11. C. 1027; Parshkov O.M. // Quant. Electron. 2018. V. 48. N 11. P. 1027.

[27] Паршков О.М. // ОПт. и спектр. 2019. Т. 126. № 24. С. 483; Parshkov O.M. // Opt. Spectrosc. 2019. V. 126. N 4. P. 400.

[28] Saleh B.A.E., Teich M.C. Fundamentals of Photonics. 2th ed. Wiley-Interscience, 2007. 1177 p.; Салех Б., Тейх М. Оптика и фотоника. Принципы и применения. Долгопрудный: ИД „Интеллект“, 2012. Т. 1.760 с.

[29] De Zafra R.L., Marshall A. // Phys. Rev. 1968. V. 170. N 1. P. 28.

[30] Физические величины. Справочник. / Под ред. Григорьева И.С., Мейлихова Е.З. М.: Энергоатомиздат, 1991. 1232 c.; Handbook of Physical Quantities. / Ed. by Grigoriev I.S., Meilikhov E.Z. Boka Raton, NY.: CRC Press, 1996. $1568 \mathrm{p}$.

[31] Mc Call S.L., Hahn E.L. // Phys. Rev. 1969. V. 183. N 2. P. 457. 